\title{
Inhibitory effects of amines from Citrus reticulata on bleomycin-induced pulmonary fibrosis in rats
}

\author{
XIAN-MEI ZHOU ${ }^{1}$, ZHEN-DONG CAO $^{2}$, NA XIAO $^{1}$, QI SHEN $^{3}$ and JIAN-XIN LI $^{3}$ \\ ${ }^{1}$ Department of Respiratory Medicine, The Affiliated Jiangsu Province Hospital of Traditional Chinese Medicine, \\ Nanjing University of Chinese Medicine, Nanjing, Jiangsu 210029; ${ }^{2}$ Department of Respiratory Medicine, \\ The Second Affiliated Jiangsu Province Hospital of Traditional Chinese Medicine, Nanjing University \\ of Chinese Medicine, Nanjing, Jiangsu 210017; ${ }^{3}$ State Key Laboratory of Analytical Chemistry \\ for Life Science and Collaborative Innovation Center of Chemistry for Life Sciences, School of Chemistry \\ and Chemical Engineering, Nanjing University, Nanjing, Jiangsu 210023, P.R. China
}

Received June 10, 2015; Accepted December 7, 2015

DOI: $10.3892 /$ ijmm.2015.2435

\begin{abstract}
Idiopathic pulmonary fibrosis (IPF) is a progressive, fatal lung disease for which, thus far, there are no effective treatments. The pericarp of Citrus reticulata, as a traditional herbal drug, has been used for the clinical treatment of lungrelated diseases in China for many years. In the present study, the amines from the pericarp of Citrus reticulata were isolated, and their hydrochlorides were prepared. The results of screening using cultured human embryonic lung fibroblasts (hELFs) revealed that, of the amines, 4-methoxyphenethylamine hydrochloride (designated as amine hydrochloride 1) possessed the most potent inhibitory effect. Further in vivo experiments using a rat model of bleomycin-induced pulmonary fibrosis demonstrated that the oral administration of amine hydrochloride 1 significantly lowered the hydroxyproline content in both serum and lung tissue, and alleviated pulmonary alveolitis and fibrosis. Immunohistochemical analysis revealed that
\end{abstract}

Correspondence to: Professor Xian-Mei Zhou, Department of Respiratory Medicine, The Affiliated Jiangsu Province Hospital of Traditional Chinese Medicine, Nanjing University of Chinese Medicine, 155 Hanzhong Road, Nanjing, Jiangsu 210029, P.R. China E-mail: zhouxianmeijs@aliyun.com

Professor Jian-Xin Li, State Key Laboratory of Analytical Chemistry for Life Science and Collaborative Innovation Center of Chemistry for Life Sciences, School of Chemistry and Chemical Engineering, Nanjing University, 163 Xianlin Ave., Nanjing, Jiangsu 210023, P.R. China

E-mail: lijxnju@nju.edu.cn

Abbreviations: IPF, idiopathic pulmonary fibrosis; hELFs, human embryonic lung fibroblasts; TGF- $\beta_{1}$, transforming growth factor- $\beta 1$; ${ }^{1} \mathrm{H}-\mathrm{NMR}$, proton nuclear magnetic resonance; MS, mass spectrum; LDH, lactate dehydrogenase; H\&E, hematoxylin and eosin; ECM, extracellular matrix

Key words: amine hydrochlorides, Citrus reticulata, idiopathic pulmonary fibrosis, inhibition, rats amine hydrochloride 1 exerted its inhibitory effect against IPF through the downregulation of lung transforming growth factor (TGF)- $\beta_{1}$ protein expression. Our results demonstrated that amine hydrochloride 1 prevented the development of bleomycin-induced lung fibrosis in rats. Thus, our data suggest that the amines from the pericarp of Citrus reticulata have therapeutic potential for use in the treatment of IPF.

\section{Introduction}

Idiopathic pulmonary fibrosis (IPF) is a chronic, progressive form of lung disease which is characterized by the abnormal and excessive deposition of collagen (fibrosis) in the pulmonary interstitium, mainly on the walls of the alveoli (1). The annual incidence of IPF was estimated at 6.8-8.8 per 100,000 individuals and $16.3-17.4$ per 100,000 individuals using narrow and broad case definitions, respectively, in the USA, and 0.22-7.4 per 100,000 individuals in Europe (2). The prognosis for patients with IPF is quite poor, and the current median survival rate following diagnosis is close to 3 years $(3,4)$. Although extensive investigations have been conducted, the cause and pathogenesis of IPF are not yet completely understood (5). However, new concepts have been recently proposed: IPF is no longer thought to be the result of inflammatory mechanisms, as previously considered, but is rather thought to be the result of a fibro-proliferative and aberrant wound healing cascade (6). Despite the increase in the number of clinical trials for IPF, the majority of the results from these trials, including those using corticosteroids, or a combination of $\mathrm{N}$-acetylcysteine, warfarin and bosentan, have been disappointing (7). Although pirfenidone and nintedanib attenuate the decline in pulmonary function, there are currently no effective pharmacological therapies to reduce the mortality rate (7-10). Thus, the discovery of novel agents with therapeutic potential for IPF is desirable.

Citrus fruits (e.g., oranges) are important fruit tree crops worldwide and are among the most commonly consumed fruits, and the global Citrus industry is worth approximately $\$ 9$ billion/year (11). The benefits of Citrus fruits are partly 
due to their phytochemical components (12), which include a wide variety of non-nutritive phytochemicals, such as flavonoids, alkaloids, anthocyanins, phenolic acids, carotenoids and tannins, together with nutritive components, such as sugars, proteins, vitamins, fibers and minerals $(13,14)$. Flavonoids, which are common polyphenols and one of the main chemical constituents of the Citrus genus, have been widely investigated for their possible role in the prevention of cardiovascular disease and cancer (15). Another important active component of Citrus fruits are adrenergic amines, such as octopamine, synephrine and tyramine, which are a type of simple alkaloid, and they exert their effects on the cardiovascular system through adrenergic stimulation $(16,17)$. Synephrine, a main constituent of Citrus fruits, stimulates lipolysis, raises the metabolic rate, and promotes the oxidation of fat, and therefore helps to reduce fat mass in obese individuals, and has been widely used in weight loss and weight management as well as in sports performance products (17). Furthermore, previous studies have revealed that the use of Citrus extract and synephrine appears to be safe, and no serious adverse effects have been directly attributed to these ingredients (17-19).

Within our research project aimed at discovering the active molecules which exert anti-fibrotic effects, we previously screened a number of Chinese herbal drugs that have been used to treat lung disease, and discovered that the pericarp of Citrus reticulata (C. reticulata) exerted inhibitory effects on pulmonary fibrosis in vitro and in vivo $(20,21)$. In traditional Chinese medicine, the pericarp of $C$. reticulata has medical functions: it regulates Qi and expels phlegm, and has been used for the treatment of lung-related diseases for a long time (22). In another previous study of ours, we revealed that the alkaline extract of $75 \%$ ethanol soluble fraction from C. reticulata was responsible for the inhibitory effects on pulmonary fibrosis in vitro and in vivo (23). Considering the chemical properties of the alkaline extract, and the alkalinity of alkaloids, we speculated that the active compounds in the alkaline extract may be the amines contained in $C$. reticulata.

As a continuation of this project, and motivated by our previous encouraging results, we conducted further research to clarify whether these amines are responsible for the inhibitory effects observed on pulmonary fibrosis. In the present study, the main amines in the alkaline extract were isolated, and their structures were determined based on nuclear magnetic resonance (NMR) and mass spectra (MS). Furthermore, the hydrochlorides of the isolated amines were prepared, and their anti-proliferative activity was assayed using a human embryonic lung fibroblast (hELF) culture system. The effects of the amine hydrochloride, which possessed potent inhibitory activity in vitro, were further evaluated using a rat model of bleomycin-induced pulmonary fibrosis, and the preliminary mechanisms were also investigated.

\section{Materials and methods}

Plant material. The pericarp of Citrus reticulata Blanco (Rutaceae) was obtained from the Department of Medicinal Materials, Affiliated Jiangsu Province Hospital of Traditional Chinese Medicine, Nanjing University of Chinese Medicine (Nanjing, China), and identified by Professor Z.N. Gong from the School of Life Science in Nanjing
Normal University (Nanjing, China). The voucher specimen (no. TCM130917) was deposited in the hospital.

Chemicals. The chemicals and reagents were purchased from the following sources: bleomycin A5 hydrochloride from Nippon Kayaku Co., Ltd. (Tokyo, Japan); prednisone from Hubei HolleyPharm Co., Ltd. (Hubei, China); hydroxyproline test kits from the Nanjing Jiancheng Bioengineering Institute (Nanjing, China); transforming growth factor (TGF)- $\beta_{1}$ from Strept Avidin-Biotin Complex (SABC); immunohistochemical test kit from Roche Diagnostics (Indianapolis, IN, USA); penicillin and streptomycin from Wuhan Boster Biological Technology, Ltd. (Wuhan, China); DMEM from HyClone (Logan, UT, USA); fetal bovine serum (FBS) from Hangzhou Ever Green Organism Engineering Materials Co., Ltd. (Hangzhou, China).

Extraction, isolation and purification. The alkaline extract of $C$. reticulata was prepared according to our previously reported procedure (23). A portion of the alkaline extract (10 g) was added to a silica gel (125 g) column, and eluted with $\mathrm{CHCl}_{3}$ triethylamine-MeOH (99.2:0.4:0.4, 98.6:0.4:1 and 97.6:0.4:2) successively to yield 5 fractions: fr-1 $(0.4 \mathrm{~g})$, fr-2 (0.6 g), fr-3 (3.9 g), fr-4 (2.5 g) and fr-5 (2.2 g). Fr-3 was further chromatographed on a medium-pressure column with $\mathrm{CHCl}_{3}$ triethylamine- $\mathrm{MeOH}$ (99.5:0.4:0.1), to obtain amines 1 (22 mg) and 2 (40 mg). Fr-4 was applied to the same medium-pressure column with $\mathrm{CHCl}_{3}$-triethylamine-MeOH (99.5:0.4:0.2) to produce compounds $2(43 \mathrm{mg})$ and $3(45 \mathrm{mg})$. Repeated medium-pressure column chromatography of fr-5 with $\mathrm{CHCl}_{3}$ triethylamine- $\mathrm{MeOH}$ (99.5:0.4:0.2) produced amines 4 (18 mg) and $5(20 \mathrm{mg})$. The structures of the isolated amines (4-methoxyphenethylamine, synephrine, para-tyramine, $N$-methyltyramine and $N$-methyl-4-methoxyphenethylamine) were determined based on detailed comparisons of proton NMR $\left({ }^{1} \mathrm{H}-\mathrm{NMR}\right)$ spectra and MS data with those of commercially available samples. For the animal experiments, the commercially available amine (Tokyo Chemical Industry, Tokyo, Japan) was used.

Preparation of amine hydrochlorides. To improve the solubility of the amines, each of the above-mentioned amines (amines 1-5) was dissolved in methanol $(5 \mathrm{mg} / \mathrm{ml})$, and $10 \% \mathrm{HCl}$ methanol solution was added $(1: 2, \mathrm{v} / \mathrm{v})$. Subsequently, the solution was completely evaporated under reduced pressure to provide each amine hydrochloride for bioassays.

Cell culture and cell viability assay. The hELFs used in the present study were obtained from the Shanghai Institutes for Biological Sciences, Chinese Academy of Sciences (Shanghai, China). The procedure for cell culture followed our previously reported method (21). In brief, the hELFs were grown in DMEM containing $10 \%$ FBS and $1 \%$ penicillin-streptomycin. The logarithmically growing cells were detached with $0.25 \%$ trypsin PBS and centrifuged $\left(100 \times \mathrm{g}, 5 \mathrm{~min}, 4^{\circ} \mathrm{C}\right)$, and the cell concentration was then adjusted to $5 \times 10^{4} / \mathrm{ml}$ using culture medium. Each amine salt was dissolved in the medium and then diluted to the desired concentrations. The amine and hELF cell solutions (each $100 \mu \mathrm{l}$ ) were added to 96-well culture plates and incubated for $48 \mathrm{~h}$. Subsequently, an MTT assay was used to evaluate cell viability. 
Lactate dehydrogenase $(L D H)$ release assay. To evaluate the cytotoxicity of the amine hydrochlorides, an LDH release assay was performed using an LDH cytotoxicity detection kit from Nanjing Keygen Biotech Co., Ltd. (Nanjing, China). hELF cell culture was conducted according to the same protocol as described above, except that the culture period was $24 \mathrm{~h}$. Following culture, the LDH concentration was measured following the manufacturer's instructions. The relative LDH release was calculated as the ratio of the $\mathrm{LDH}$ release over the positive control, and the positive control was treated with only $1 \%$ NP-40 and set as $100 \%$ LDH release, as previously reported (23). All cultures were kept in a $\mathrm{CO}_{2}$ incubator under moist conditions with $5 \% \mathrm{CO}_{2}$, at $37^{\circ} \mathrm{C}$.

Animals. The rats used in the present study were purchased from the Laboratory Animal Center, Nanjing University of Chinese Medicine (Nanjing, China). Pathogen-free mature male Sprague-Dawley (SD) rats (weighing 180-200 g) were used in our experiments. The rats were housed in a conventional animal facility with a 12-h light/dark cycle, and had access to standard pelleted food and water ad libitum. The rats were allowed to acclimatize for 7 days prior to their use in our experiments.

All of the procedures involving animals and their care were approved by the Jiangsu Animal Care and Use Committee and followed the national and institutional rules regarding animal experiments.

Model of bleomycin-induced pulmonary fibrosis. The SD rats were randomly divided into 6 groups ( $\mathrm{n}=6$ per group). The rat model of bleomycin-induced pulmonary fibrosis was established following our previously reported method (23). Briefly, the rats were anesthetized by an intraperitoneal injection of pentobarbital, followed by a single intratracheal instillation of $5 \mathrm{mg} / \mathrm{kg}$ of bleomycin in $2.0 \mathrm{ml} / \mathrm{kg}$ PBS. The animals in the normal group received an intratracheal injection of an equal volume of the PBS instead of bleomycin. There were 4 groups for drug treatment: the rats were orally administered 4-methoxyphenethylamine hydrochloride (designated as amine hydrochloride 1) once daily at doses of 5 (treatment group 1; 1-5), 10 (treatment group 2; 1-10) and 20 (treatment group 3;1-20) $\mathrm{mg} / \mathrm{kg}$ (in distilled water) and prednisone (treatment group 4) at a dose of $5 \mathrm{mg} / \mathrm{kg}$ once per day. The animals in the normal (group 5) and control (group 6) groups received an equal volume of distilled water only. The experimental period lasted for 4 weeks. At the end of the experiment, the rats were euthanized by pentobarbital administration, and the serum and lung tissues were collected for bioassays. The blood was centrifuged at 2,500 rpm for $20 \mathrm{~min}$, and then kept at $-20^{\circ} \mathrm{C}$ until use. The lung vasculature was perfused to free the blood. The right lung was fixed in neutral buffered $10 \%$ formalin $(\mathrm{pH}$ 7.4) for histopathological analysis and analysis of TGF- $\beta 1$ expression. The left lung was frozen in liquid nitrogen and stored at $-80^{\circ} \mathrm{C}$ for hydroxyproline level measurements.

Histopathological evaluation of lung tissues. The right lung tissue was fixed by using $10 \%$ neutralized buffered formalin on the trachea, and embedded in paraffin. Serial sections (4- $\mu$ m-thick) were acquired and stained with hematoxylin and eosin (H\&E) and Masson's trichrome staining, following the manufacturer's instructions for light microscopic evaluation. The histological grading of alveolitis and fibrosis in the lung specimens was performed by experienced pathologists in a blinded manner, and recorded in grades from - to +++ and corresponding scores from 0 to 3 , as previously described (24).

Assay for hydroxyproline in serum and lungs. The hydroxyproline content was determined as an index of the collagen content in the serum and left lung tissues as previously described (20). Briefly, $0.5 \mathrm{ml}$ of serum or 30-100 mg lung tissue samples were respectively hydrolyzed in $1 \mathrm{ml}$ lysis buffer solution $(\mathrm{pH} 7.4,10 \mathrm{mM}$ Tris-HCl, $0.1 \mathrm{mM}$ EDTA-2Na, $10 \mathrm{mM}$ saccharose, $0.8 \%$ sodium chloride solution) at $100^{\circ} \mathrm{C}$ for $20 \mathrm{~min}$. Hydroxyproline was then measured using the test kit according to the manufacturer's instructions. The absorbance of colored products was measured at $550 \mathrm{~nm}$.

$T G F-\beta_{1}$ protein expression assay. The semi-quantification of the TGF- $\beta_{1}$ protein levels in the right lungs of rats was assessed with an SABC immunohistochemical test kit following the manufacturer's instructions as previously described (20). Briefly, paraffin-embedded lung sections were dewaxed with xylene and dehydrated with a series of ethanol. Endogenous peroxidase was inactivated by treatment with $3 \%$ hydrogen peroxide $\left(\mathrm{H}_{2} \mathrm{O}_{2}\right)$ for $10 \mathrm{~min}$ at room temperature. Non-specific binding was blocked with normal goat serum for $20 \mathrm{~min}$. The sections were then incubated with primary antibody rabbit anti-TGF- $\beta 1$ for $2 \mathrm{~h}$ at $37^{\circ} \mathrm{C}$, and successively with biotinylated goat anti-rabbit secondary antibody for $20 \mathrm{~min}$ at $25^{\circ} \mathrm{C}$, followed by SABC for $20 \mathrm{~min}$ at $25^{\circ} \mathrm{C}$. Immunoreactivity was detected by the addition of diaminobenzidine (DAB). The sections were counterstained with hematoxylin, dehydrated and mounted with permont. As the negative controls, each primary antibody was substituted with PBS. The protein expression of TGF- $\beta 1$ was assessed using a quantitative image analysis system.

Statistical analysis. Data are presented as the means \pm standard deviation (SD). Statistical analysis was undertaken by analysis of variance (ANOVA) followed by appropriate post hoc tests, including multiple comparison tests (LSD and t-test). The alveolitis and fibrosis scores of lung tissues were evaluated using the Mann-Whitney test. The SPSS 13.0 software package (SPSS, Inc., Chicago, IL, USA) was used for the analysis. A p-value $<0.05$ was considered to indicate a statistically significant difference.

\section{Results}

Amines and their hydrochloride preparations. The alkaline extract of $C$. reticulata $(10 \mathrm{~g})$ was first subjected to normaland medium-pressure silica gel column chromatographies repeatedly, and subsequently 5 amines, 4-methoxyphenethylamine, synephrine, para-tyramine, $N$-methyltyramine and $N$-methyl-4-methoxyphenethylamine were isolated. The structures of the isolated amines were then determined, based on detailed comparisons of ${ }^{1} \mathrm{H}-\mathrm{NMR}$ spectra and MS data with those of commercially available samples (data not shown).

To improve the aqueous solubility of the isolated amines, the amine hydrochlorides, 4-methoxyphenethylamine hydrochloride (amine hydrochloride 1), synephrine hydrochloride (amine hydrochloride 2), para-tyramine hydrochloride (amine hydrochloride 3), $N$-methyltyramine hydrochloride (amine 
Table I. Effects of amine hydrochloride 1 and bleomycin on the body weight of rats.

\begin{tabular}{llllll}
\hline Group & Day 0 & Day 7 & Day 14 & Day 21 & Day 28 \\
\hline Normal & $202.1 \pm 14.5$ & $236.2 \pm 5.1$ & $246.7 \pm 20.8$ & $290.2 \pm 30.9$ & $306.5 \pm 40.1$ \\
Control & $201.8 \pm 10.5$ & $195.3 \pm 7.9^{\mathrm{a}}$ & $210.2 \pm 27.3^{\mathrm{a}}$ & $225.8 \pm 29.9^{\mathrm{a}}$ & $245.3 \pm 33.4^{\mathrm{a}}$ \\
Prednisone & $190.3 \pm 14.7$ & $213.1 \pm 12.8^{\mathrm{b}}$ & $219.7 \pm 27.1$ & $228.2 \pm 26.6$ & $240.2 \pm 38.7$ \\
$1-5$ & $195.6 \pm 13.4$ & $205.4 \pm 7.9$ & $218.6 \pm 25.0$ & $239.5 \pm 27.3$ & $246.1 \pm 41.6$ \\
$1-10$ & $199.2 \pm 9.5$ & $207.4 \pm 4.7^{\mathrm{b}}$ & $248.0 \pm 20.3^{\mathrm{c}}$ & $286.0 \pm 28.1^{\mathrm{c}}$ & $297.1 \pm 44.8^{\mathrm{c}}$ \\
$1-20$ & $201.3 \pm 13.6$ & $198.4 \pm 3.2$ & $238.3 \pm 24.8^{\mathrm{b}}$ & $276.1 \pm 24.2^{\mathrm{c}}$ & $294.0 \pm 34.2^{\mathrm{c}}$
\end{tabular}

Normal group, saline + water; control group, bleomycin + water; prednisone group, bleomycin + prednisone at a dose of $5 \mathrm{mg} / \mathrm{kg}$ once per day. Groups 1-5, 1-10 and 1-20, bleomycin + 4-methoxyphenethylamine hydrochloride (amine hydrochloride 1) at doses of 5, 10 and $20 \mathrm{mg} / \mathrm{kg}$ once per $\mathrm{da}$, respectively. Data are expressed as the means $\pm \mathrm{SD}, \mathrm{n}=6$ animals per group. ${ }^{\mathrm{a}} \mathrm{p}<0.01$ vs. normal group; ${ }^{\mathrm{b}} \mathrm{p}<0.05$ and ${ }^{\mathrm{c}} \mathrm{p}<0.01 \mathrm{vs}$. control group.

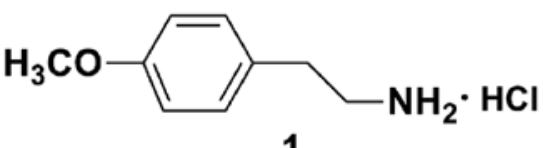

1

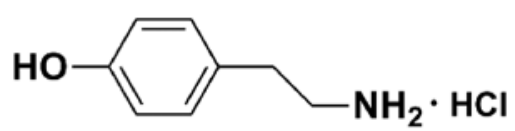

3

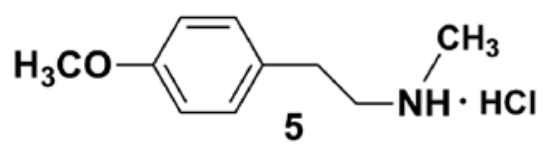<smiles>CNCC(O)c1ccc(O)cc1</smiles>

2<smiles>CNCCc1ccc(O)cc1</smiles>

4

Figure 1. Chemical structures of amine hydrochlorides 1 (4-methoxyphenethylamine hydrochloride), 2 (synephrine hydrochloride), 3 (para-tyramine hydrochloride), 4 ( $N$-methyltyramine hydrochloride) and 5 ( $N$-methyl-4-methoxyphenethylamine hydrochloride).

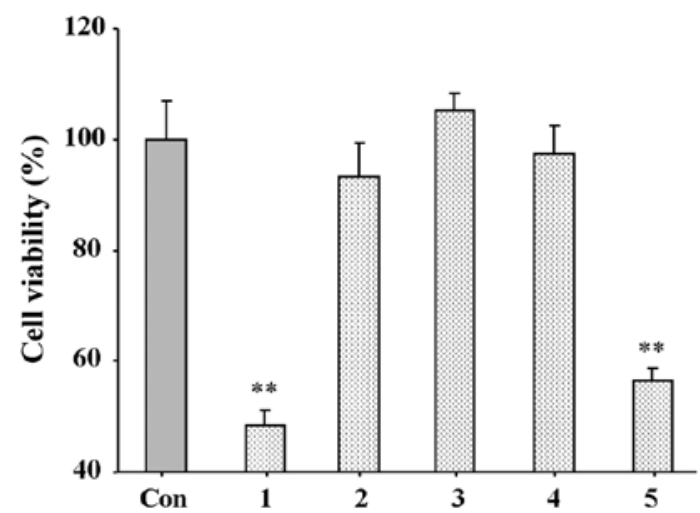

Figure 2. Effect of amine hydrochlorides on the proliferation of human embryonic lung fibroblasts (hELFs). The data of the control group (Con) were pegged as $100.0 \%$, whereas other data were calculated relative to it. Values are expressed as the means $\pm \mathrm{SD}, \mathrm{n}=5$ wells per group. ${ }^{* *} \mathrm{p}<0.01$ vs. control group.

hydrochloride 4) and $N$-methyl-4-methoxyphenethylamine hydrochloride (amine hydrochloride 5) were prepared with $10 \% \mathrm{HCl}$ methanol solution (Fig. 1), and used for all of the following bioassays.

Inhibitory effects and cytotoxicity to hELFs. To screen the inhibitory activity of the amine hydrochlorides, hELFs were used. All amine hydrochlorides were dissolved in medium, and their inhibitory activity on the proliferation of hELFs was firstly assayed at $10 \mu \mathrm{M}$. As shown in Fig. 2, amine hydrochlorides 1 and 5 significantly inhibited hELF proliferation following $48 \mathrm{~h}$ of incubation, and it should be noted that amine hydrochloride 1 caused a $51.63 \%$ inhibition of cell viability. Amine hydrochloride 3 exerted no inhibitory effect, whereas amine hydrochlorides 2 and 4 exerted a weak inhibitory effect. Due to the fact that compound 1 displayed the most potent effect, further detailed tests on its minimal half inhibitory concentration $\left(\mathrm{IC}_{50}\right)$ were performed, and the $\mathrm{IC}_{50}$ of compound 1 was noted as $12.7 \mu \mathrm{M}$.

The cytotoxicity of amine hydrochlorides 1 and 5 on hELF viability was evaluated by an $\mathrm{LDH}$ release assay. The results of the assay revealed that at concentrations up to $20 \mu \mathrm{M}$, amine hydrochlorides 1 and 5 did not cause cytotoxicity to the cells (data not shown).

Effects of amine hydrochloride 1 on bleomycin-induced weight loss in rats. As shown in Table I, the body weight of the rats decreased significantly on the 7 th day following the administration of bleomycin and decreased gradually thereafter throughout the whole experiment period compared to the normal rats $(p<0.01)$. Even though the rats in the control group gained weight slightly throughout the experimental 


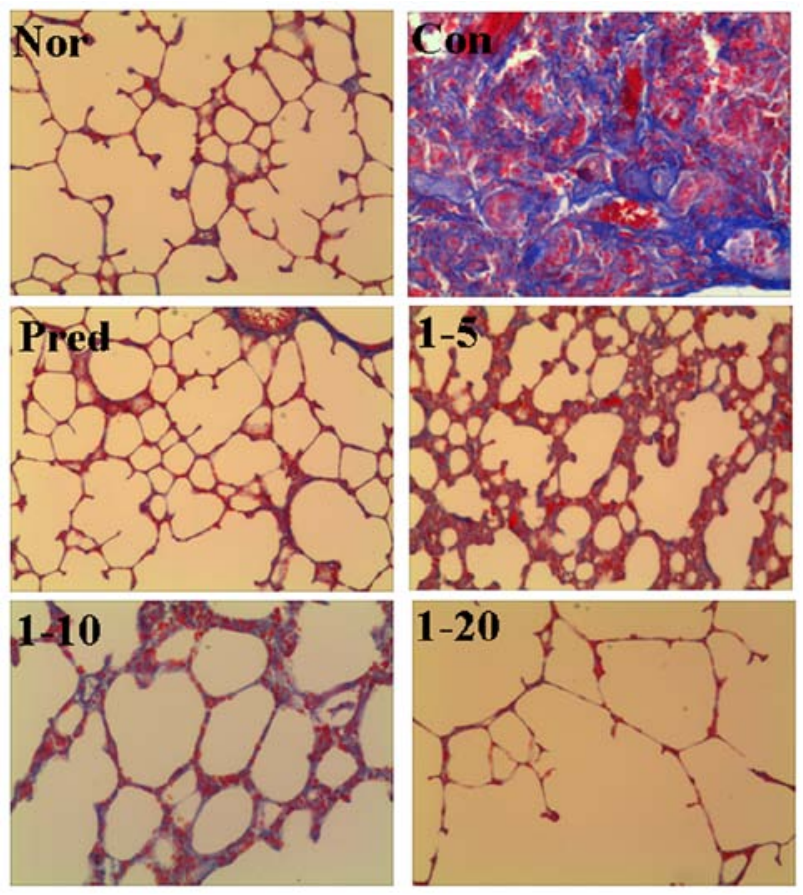

Figure 3. Effect of 4-methoxyphenethylamine hydrochloride (amine hydrochloride 1) on collagen accumulation induced by bleomycin. Masson's trichrome staining was performed on the lung tissues collected on day 28 after the administration of bleomycin. All photomicrographs were taken at x100 magnification using a microscope. Collagen deposition was stained in blue. Normal (Nor) and control (Con) groups received distilled water as treatment. Rats in the prednisone group (Pred) were treated with prednisone at $5 \mathrm{mg} / \mathrm{kg} / \mathrm{day}$. Rats in groups 1-5, 1-10 and 1-20 were treated with amine hydrochloride 1 at doses of 5,10 and $20 \mathrm{mg} / \mathrm{kg} /$ day, respectively.

period, their weight was lower than the rats in the normal group. Prednisone, a clinically available drug used to treat IPF was used as a positive control, did not have much of a positive impact on weight loss. However, body weight was significantly increased following treatment with amine hydrochloride 1 at doses of 10 and $20 \mathrm{mg} / \mathrm{kg} / \mathrm{day}$ from the 14th to 28th day compared to the bleomycin-treated group.

Effects of amine hydrochloride 1 on bleomycin-induced collagen deposition and hydroxyproline expression in rats. The effects of amine hydrochloride 1 on collagen deposition in the rats with IPF were first evaluated by light microscopy using lung tissue sections stained with Masson's trichrome staining. As shown in Fig. 3, in the normal group, only a small amount of collagen fibers in the alveolar septum were observed. In the lungs of the bleomycin-treated rats, we noted an extended web of collagen-positive stained areas in an irregular pattern (control group). However, the lungs of the rats treated with amine hydrochloride 1 displayed less collagen accumulation compared with those of the rats in the control group, particularly in the rats treated with amine hydrochloride 1 at $20 \mathrm{mg} / \mathrm{kg} / \mathrm{day}$, where only a mild deposition of collagen fibers in the alveolar septum was observed.

Subsequently, the hydroxyproline content in the lungs and serum was determined. The rats treated with bleomycin alone (the control group) showed a significant increase in hydroxyproline content, by 1.8 -fold in the lungs $(\mu \mathrm{g} / \mathrm{g}, \mathrm{p}<0.01)$ and 1.36 -fold in the serum $(\mu \mathrm{g} / \mathrm{ml}, \mathrm{p}<0.01)$, compared with those
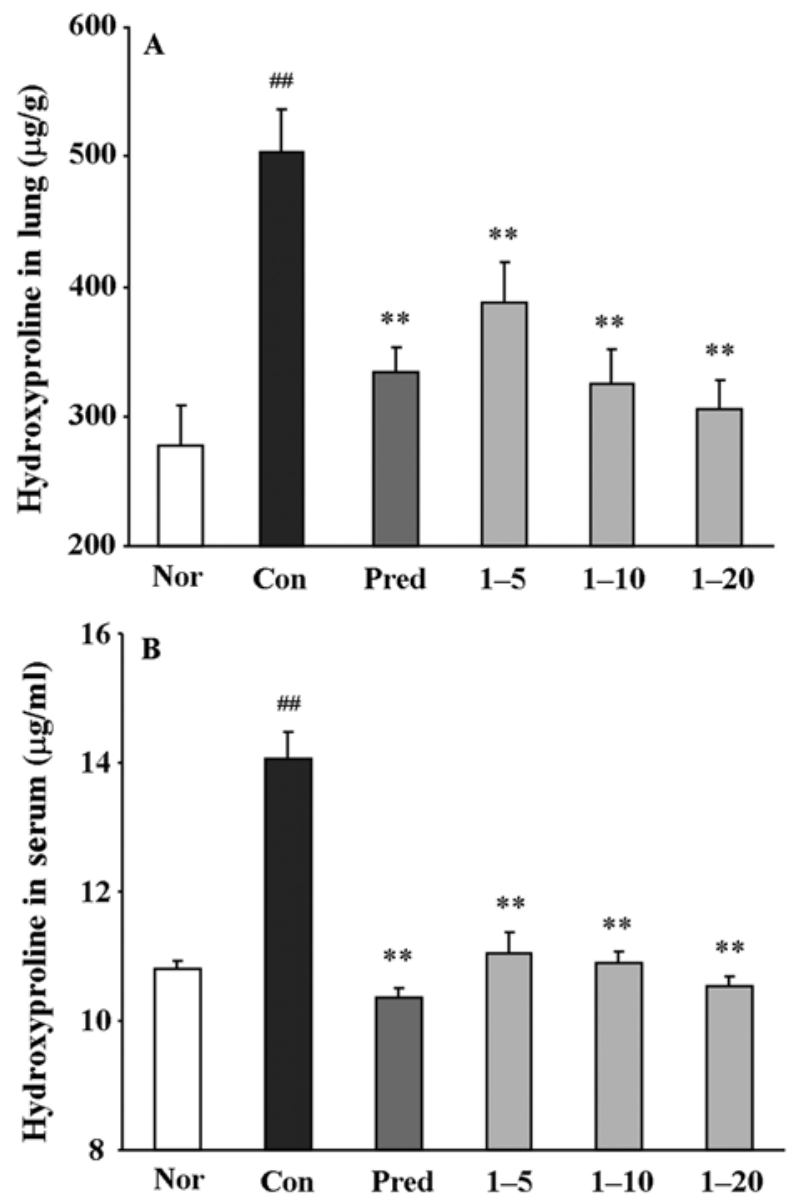

Figure 4. Effect of 4-methoxyphenethylamine hydrochloride (amine hydrochloride 1) on the hydroxyproline content in (A) lung tissues and (B) serum. Normal (Nor) and control (Con) groups received distilled water as treatment. The rats in the prednisone group (Pred) were treated with prednisone at $5 \mathrm{mg} /$ $\mathrm{kg} /$ day. Rats in groups 1-5, 1-10 and 1-20 were treated with amine hydrochloride 1 at doses of 5,10 and $20 \mathrm{mg} / \mathrm{kg} /$ day, respectively. Values are expressed as the means $\pm \mathrm{SD}, \mathrm{n}=6$ animals per group. ${ }^{\# \#} \mathrm{p}<0.01$ vs. normal group; ${ }^{* *} \mathrm{p}<0.01$ vs. control group.

of normal rats (Fig. 4). Treatment with amine hydrochloride 1 at all doses significantly decreased the hydroxyproline contents in both the lung and serum compared with the control group. These results clearly indicated that amine hydrochloride 1 inhibited collagen deposition.

Effect of amine hydrochloride 1 on bleomycin-induced pathological changes. Lung fibrosis was further scored by histopathological observations of the lung sections on the 28th day. H\&E staining of the lung tissues from the normal rats revealed a normal histological appearance in terms of bronchi, bronchioles and alveoli (Fig. 5, Nor). The administration of bleomycin caused increased thickness of the alveolar wall, alveolar and vascular congestion, and severe infiltration of inflammatory cells in the alveolar septa and interstitium in lung tissues were observed (Fig. 5, Con). The prednisone group displayed only slightly thickened alveolar walls with some inflammatory cells. Treatment with amine hydrochloride 1 significantly reduced bleomycin-induced inflammatory cell infiltration, ameliorated the thickening of the interalveolar septum with edema and restored the alveolar architecture (Fig. 5, 1-5, 1-10 and 1-20). 


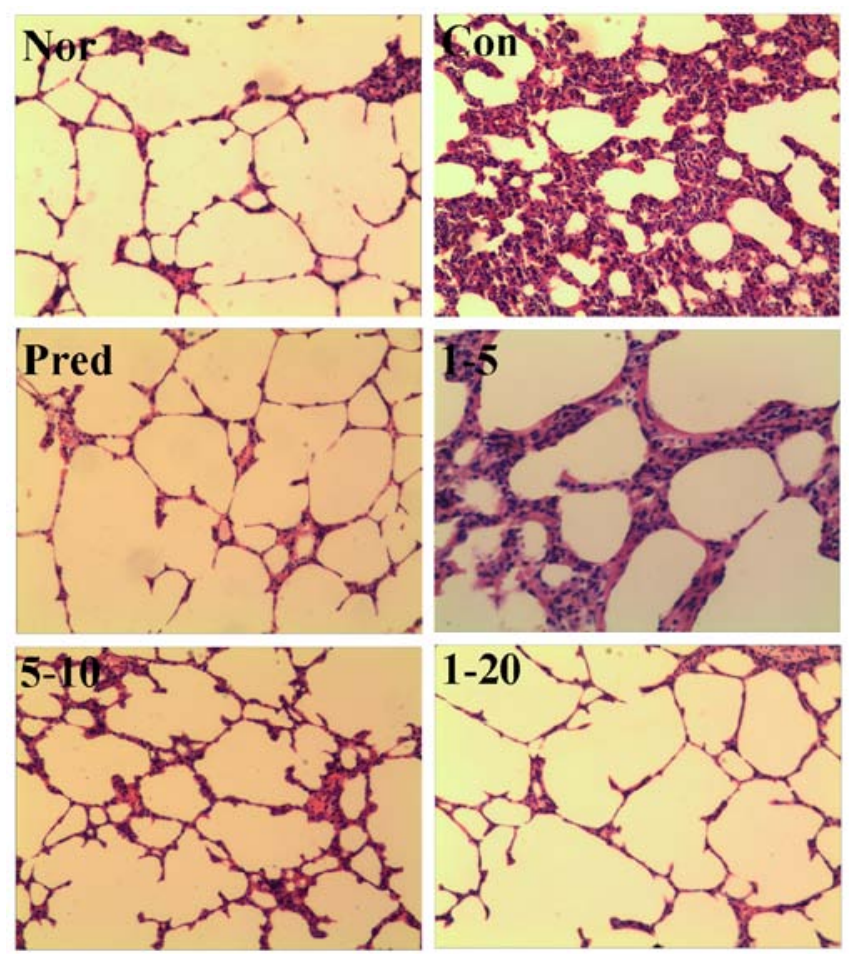

Figure 5. Effect of 4-methoxyphenethylamine hydrochloride (amine hydrochloride 1) on alveolitis and fibrosis. H\&E staining was performed on the lung tissues collected on day 28 after the administration of bleomycin. All photomicrographs were taken at x100 magnification using a microscope. Rats in the normal (Nor) and control (Con) groups were treated with distilled water. Rats in the prednisone group (Pred) were treated with prednisone at $5 \mathrm{mg} / \mathrm{kg} / \mathrm{day}$. Rats in groups 1-5, 1-10 and 1-20 were treated with amine hydrochloride 1 at doses of 5,10 and $20 \mathrm{mg} / \mathrm{kg} /$ day, respectively.

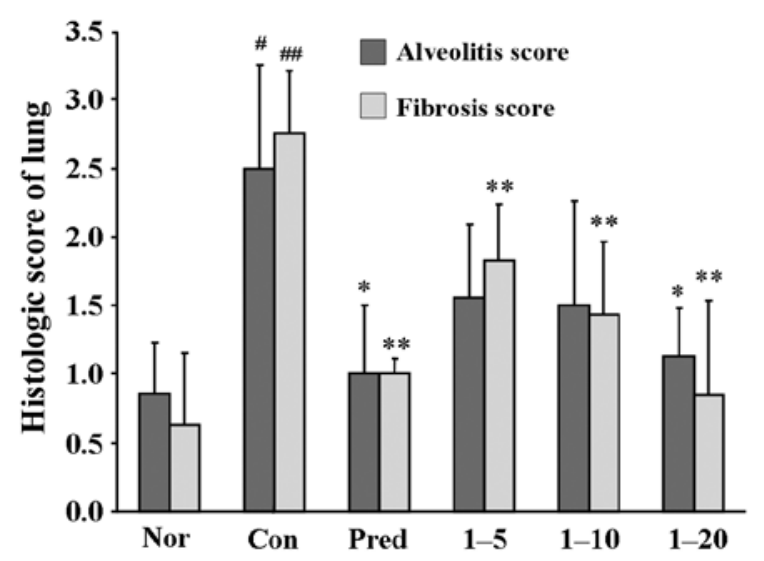

Figure 6. Effect of 4-methoxyphenethylamine hydrochloride (amine hydrochloride 1) on alveolitis and fibrosis. Rats in the normal (Nor) and control (Con) groups were treated with distilled water. Rats in the prednisone group (Pred) was treated with prednisone at $5 \mathrm{mg} / \mathrm{kg} /$ day. Rats in groups $1-5,1-10$ and 1-20 were treated with amine hydrochloride 1 at doses of 5,10 and $20 \mathrm{mg} / \mathrm{kg} / \mathrm{day}$, respectively. Values are expressed as the means $\pm \mathrm{SD}, \mathrm{n}=6$ animals per group. ${ }^{\#} \mathrm{p}<0.05$ and ${ }^{\# \#} \mathrm{p}<0.01$ vs. normal group; ${ }^{*} \mathrm{p}<0.05$ and $^{* *} \mathrm{p}<0.01$ vs. control group.

As shown in Fig. 6, the semi-quantitative assessment of the lung sections revealed that an approximately 3 -fold score increase in alveolitis, and 4.4-fold in fibrosis, was observed in the control groups compared to the normal group. Both the alveolitis and fibrosis scores were significantly decreased in the
Table II. Effects of amine hydrochloride 1 and bleomycin on TGF- $\beta_{1}$ expression in lung tissues.

\begin{tabular}{lll}
\hline Group & Area $\left(\mu \mathrm{m}^{2}\right)$ & Integral density \\
\hline Normal & $249.09 \pm 44.92$ & $19.20 \pm 10.23$ \\
Control & $450.62 \pm 56.21^{\mathrm{a}}$ & $86.43 \pm 11.04^{\mathrm{a}}$ \\
Prednisone & $306.27 \pm 43.63^{\mathrm{b}}$ & $20.03 \pm 9.02^{\mathrm{b}}$ \\
$1-5$ & $357.66 \pm 52.19^{\mathrm{b}}$ & $64.23 \pm 8.94^{\mathrm{b}}$ \\
$1-10$ & $330.57 \pm 67.82^{\mathrm{b}}$ & $45.98 \pm 11.02^{\mathrm{b}}$ \\
$1-20$ & $268.34 \pm 62.24^{\mathrm{b}}$ & $21.01 \pm 10.52^{\mathrm{b}}$ \\
\hline
\end{tabular}

Normal group, saline + water; control group, bleomycin + water' prednisone group, bleomycin + prednisone at a dose of $5 \mathrm{mg} / \mathrm{kg}$ once per day. Groups 1-5, 1-10 and 1-20, bleomycin + 4-methoxyphenethylamine hydrochloride (amine hydrochloride 1) at doses of 5,10 and $20 \mathrm{mg} /$ $\mathrm{kg}$ once per day, respectively. Data are expressed as the means $\pm \mathrm{SD}$, $\mathrm{n}=6$ animals per group. ${ }^{\mathrm{a}} \mathrm{p}<0.01 \mathrm{vs}$. normal group; ${ }^{\mathrm{b}} \mathrm{p}<0.01 \mathrm{vs}$. control group. TGF- $\beta_{1}$, transforming growth factor $-\beta_{1}$.

prednisone-treated groups. The lungs of the rats treated with amine hydrochloride 1 only at $20 \mathrm{mg} / \mathrm{kg} /$ day exhibited reduced pathological alveolitis scores, and the fibrosis scores were markedly attenuated at all doses.

Effect of amine hydrochloride 1 on bleomycin-induced TGF- $\beta_{1}$ protein expression. Since TGF- $\beta_{1}$ appears to be closely associated with fibroblast-to-myofibroblast activation and drives pulmonary fibrosis in the current rat model (21), TGF- $\beta_{1}$ protein expression was assessed using immunohistochemical staining. The results of the protein expression of TGF- $\beta_{1}$ in the lungs is shown in Table II. As expected, the administration of bleomycin markedly induced TGF- $\beta_{1}$ protein expression, while the positive control, prednisone, significantly suppressed its expression. Treatment of the rats with amine hydrochloride 1 significantly repressed TGF- $\beta_{1}$ protein overexpression at all doses, particularly the dose of $20 \mathrm{mg} / \mathrm{kg} / \mathrm{day}$, which decreased the expression to values close to those of the normal group.

\section{Discussion}

As described in a previous study of ours, the alkaline extract from the pericarp of $C$. reticulata exerted inhibitory effects on pulmonary fibrosis in vitro and in vivo (23). The amine possesses alkalinity, and thus the chemical properties of the alkaline extract, and elucidation of isolation and its structure, focusing on amine components, were performed. As a result, 5 small amines were obtained from the alkaline extract. As the amines possessed poor solubility in aqueous solution, to improve their solubility, their hydrochlorides were prepared.

Fibroblasts are mesenchymal cells which are derived from embryonic mesodermal tissue, and their activation plays a vital role in wound healing (25). One of the key characteristics of IPF is a dysregulated wound-healing response that leads to the fatal accumulation of fibroblasts in the lungs, and expansion of the fibroblast population with the development of fibrotic lesions known as fibroblast 'foci' in the lungs is believed to contribute to the progression of fibrosis (26). The inhibition of lung fibroblast differentiation thus has potential therapeutic benefits for 
IPF. Therefore, in the present study, hELFs were used to screen the inhibitory activity of the amine hydrochlorides. The results indicated that amine hydrochlorides 1 and 5 exerted inhibitory effects, whereas others exerted almost no effect. On the basis of these data, we suggest that the methoxyl group, at the benzene ring of the amines, is essential for this activity.

To verify that the inhibitory activity of amine hydrochloride 1 and 5 on hELF was not due to its cytotoxicity, the effects of the amines on cell viability were evaluated by an LDH release assay. The data demonstrated that the inhibitory activity was not due to their cytotoxicity.

To better understand the anti-fibrotic effects of the tested compounds and the pathogenesis of lung fibrosis disorders, multiple animal models have been developed, such as silica-, radiation-induced fibrosis (27). Among currently applied models of experimentally induced pulmonary fibrosis, the administration of bleomycin is the most commonly used in studies on experimental lung fibrosis (27). Furthermore, the administration of bleomycin resulted in notable weight loss. Fibroblast proliferation and extracellular matrix collagen deposition constitute one of the key factors of IPF development, and accumulation of the newly formed collagen in the lung interstitium leads to the thickening of the alveolar septum and lung dysfunction. Thus, the level of collagen is a hallmark of bleomycin-induced IPF (28). Since collagen contains a high ratio of hydroxyproline, the estimation of the total hydroxyproline content is widely considered a reliable index for collagen deposition (28). Therefore, both of them are necessary to reflect collagen accumulation in IPF. The delivery of bleomycin via the intratracheal route also results in a direct cell injury via DNA damage (27). Subsequently, histopathological abnormalities, such as a thickened alveolar wall, collapsed alveolar spaces and focal honeycombing, infiltration of inflammatory cells are observed $(27,29)$. In the present study, amine hydrochloride 1 significantly increased the rat body mass and lowered the hydroxyproline content in both lung tissue and serum, which revealed that amine hydrochloride 1 exerted an inhibitory effect on collagen deposition. In terms of the pathological changes to the lungs, amine hydrochloride 1 markedly improved the scores for alveolitis and fibrosis, and inhibited lung inflammation. These results clearly demonstrate that amine hydrochloride 1 suppresses both the acute inflammatory response and fibrogenic changes that are key factors of IPF.

Although multiple signaling pathways are currently known to be involved in the development and progression of IPF, the TGF- $\beta$ family comprises multifunctional cytokines which regulate cell growth, apoptosis, inflammation and extracellular matrix (ECM) synthesis (30). The TGF- $\beta$ signaling pathway plays important roles in wound healing and organ fibrosis, depending on the cellular context (30). Moreover, TGF- $\beta_{1}$ appears to be the most prevalent isoform, which is considered as a key pro-fibrotic agent since it stimulates extracellular matrix production, fibroblast-to-myofibroblast differentiation and inhibition of autophagy in fibroblasts $(31,32)$. In the present study, on the 28th day following the administration of bleomycin, the protein level of TGF- $\beta_{1}$ was clearly increased, while treatment with amine hydrochloride 1 inhibited the overexpression of TGF- $\beta_{1}$ protein. These results suggest that the anti-pulmonary fibrotic effect of amine hydrochloride 1 should be ascribed, at least partially, to the downregulation of the protein expression of TGF- $\beta_{1}$.
In conclusion, the findings of the present study demonstrate that amine hydrochloride 1 is a highly promising agent which can be used for the prevention of lung inflammation and fibrosis, and the anti-fibrotic effects seem to be mediated, at least partially, through the inhibition of TGF- $\beta_{1}$ protein expression. The present results also revealed that the amines from the pericarp of Citrus fruits have therapeutic potential for the prevention of IPF. Further studies are required to clarify the detailed mechanisms of action of Citrus fruits, and are currently being undertaken in our laboratory.

\section{Acknowledgements}

The present study was supported by the Key Discipline of Jiangsu Province Administration of Traditional Chinese Medicine (JS1302).

\section{References}

1. Raghu G, Collard HR, Egan JJ, Martinez FJ, Behr J, Brown KK, Colby TV, Cordier JF, Flaherty KR, Lasky JA, et al; ATS/ERS/JRS/ALAT Committee on Idiopathic Pulmonary Fibrosis: An official ATS/ERS/JRS/ALAT statement: idiopathic pulmonary fibrosis: evidence-based guidelines for diagnosis and management. Am J Respir Crit Care Med 183: 788-824, 2011.

2. Nalysnyk L, Cid-Ruzafa J, Rotella P and Esser D: Incidence and prevalence of idiopathic pulmonary fibrosis: review of the literature. Eur Respir Rev 21: 355-361, 2012.

3. Hilberg O, Simonsen U, du Bois R and Bendstrup E: Pirfenidone: significant treatment effects in idiopathic pulmonary fibrosis. Clin Respir J 6: 131-143, 2012.

4. O'Connell OJ, Kennedy MP and Henry MT: Idiopathic pulmonary fibrosis: treatment update. Adv Ther 28: 986-999, 2011.

5. Armanios M: Telomerase and idiopathic pulmonary fibrosis. Mutat Res 730: 52-58, 2012.

6. Rafii R, Juarez MM, Albertson TE and Chan AL: A review of current and novel therapies for idiopathic pulmonary fibrosis. J Thorac Dis 5: 48-73, 2013.

7. Spagnolo P, Maher TM and Richeldi L: Idiopathic pulmonary fibrosis: recent advances on pharmacological therapy. Pharmacol Ther 152: 18-27, 2015.

8. Richeldi L, du Bois RM, Raghu G, Azuma A, Brown KK, Costabel U, Cottin V, Flaherty KR, Hansell DM, Inoue Y, et al; INPULSIS Trial Investigators: efficacy and safety of nintedanib in idiopathic pulmonary fibrosis. N Engl J Med 370: 2071-2082, 2014.

9. King TE Jr, Bradford WZ, Castro-Bernardini S, Fagan EA, Glaspole I, Glassberg MK, Gorina E, Hopkins PM, Kardatzke D, Lancaster L, et al; ASCEND Study Group: A phase 3 trial of pirfenidone in patients with idiopathic pulmonary fibrosis. N Engl J Med 370: 2083-2092, 2014.

10. Cottin V: Current approaches to the diagnosis and treatment of idiopathic pulmonary fibrosis in Europe: the AIR survey. Eur Respir Rev 23: 225-230, 2014.

11. Velasco R and Licciardello C: A genealogy of the citrus family. Nat Biotechnol 32: 640-642, 2014

12. Medina S, Ferreres F, García-Viguera C, Horcajada MN, Orduna J, Savirón M, Zurek G, Martínez-Sanz JM, Gil JI and Gil-Izquierdo A: Non-targeted metabolomic approach reveals urinary metabolites linked to steroid biosynthesis pathway after ingestion of citrus juice. Food Chem 136: 938-946, 2013.

13. Nile SH and Park SW: Bioactive components and healthpromoting properties of Yuzu (Citrus ichangensis x C.reticulate). Food Rev Int 30: 155-167, 2014.

14. Yadav L and Kalidhar SB: Chemical components of Mandarin species: a review. J Indian Chem Soc 88: 911-926, 2011.

15. Khan MK, Huma ZE and Dangles O: A comprehensive review on flavanones, the major citrus polyphenols. J Food Compos Anal 33: 85-104, 2014.

16. Vieira SM, Theodoro KH and Glória MBA: Profile and levels of bioactive amines in orange juice and orange soft drink. Food Chem 100: 895-903, 2007.

17. Stohs SJ, Preuss HG and Shara M: The safety of Citrus aurantium (bitter orange) and its primary protoalkaloid p-synephrine. Phytother Res 25: 1421-1428, 2011. 
18. Kaats GR, Miller H, Preuss HG and Stohs SJ: A 60day doubleblind, placebo-controlled safety study involving Citrus aurantium (bitter orange) extract. Food Chem Toxicol 55: 358-362, 2013.

19. Stohs SJ and Preuss HG: Stereochemical and pharmacological differences between naturally occurring p-synephrine and synthetic p-synephrine. J Funct Foods 4: 2-5, 2012.

20. Zhou XM, Zhang GC, Li JX and Hou J: Inhibitory effects of Hu-qi-yin on the bleomycin-induced pulmonary fibrosis in rats. J Ethnopharmacol 111: 255-264, 2007.

21. Zhou XM, Huang MM, He CC and Li JX: Inhibitory effects of citrus extracts on the experimental pulmonary fibrosis. J Ethnopharmacol 126: 143-148, 2009.

22. China Pharmacopoeia Committee: Citri Reticulatae Pericarpium In: Chinese Pharmacopeia 2010. Chemical Industry Press, Beijing, pp176-177, 2010.

23. Zhou XM, Wen GY, Zhao Y, Liu YM and Li JX: Inhibitory effects of alkaline extract of Citrus reticulata on pulmonary fibrosis. J Ethnopharmacol 146: 372-378, 2013.

24. Szapiel SV, Elson NA, Fulmer JD, Hunninghake GW and Crystal RG: Bleomycin-induced interstitial pulmonary disease in the nude, athymic mouse. Am Rev Respir Dis 120: 893-899, 1979.

25. Camelo A, Dunmore R, Sleeman MA and Clarke DL: The epithelium in idiopathic pulmonary fibrosis: breaking the barrier. Front Pharmacol 4: 173, 2014.
26. Kendall RT and Feghali-Bostwick CA: Fibroblasts in fibrosis: novel roles and mediators. Front Pharmacol 5: 123, 2014.

27. B Moore B, Lawson WE, Oury TD, Sisson TH, Raghavendran K and Hogaboam CM: Animal models of fibrotic lung disease. Am J Respir Cell Mol Biol 49: 167-179, 2013.

28. Kliment CR, Englert JM, Crum LP and Oury TD: A novel method for accurate collagen and biochemical assessment of pulmonary tissue utilizing one animal. Int J Clin Exp Pathol 4: 349-355, 2011.

29. Huaux F, Noel S, Dhooghe B, Panin N, Lo Re S, Lison D, Wallemacq P, Marbaix E, Scholte BJ, Lebecque P and Leal T: Dysregulated proinflammatory and fibrogenic phenotype of fibroblasts in cystic fibrosis. PLoS One 8: e64341, 2013.

30. Yan Z, Kui Z and Ping Z: Reviews and prospectives of signaling pathway analysis in idiopathic pulmonary fibrosis. Autoimmun Rev 13: 1020-1025, 2014.

31. Klingberg F, Hinz B and White ES: The myofibroblast matrix: implications for tissue repair and fibrosis. J Pathol 229: 298-309, 2013.

32. Della Latta V, Cecchettini A, Del Ry S and Morales MA Bleomycin in the setting of lung fibrosis induction: from biological mechanisms to counteractions. Pharmacol Res 97: 122-130, 2015. 